\title{
New Recursive Approximations for Variable-Order Fractional Operators with Applications
}

\section{Mahmoud A. Zaky ${ }^{a}$, Eid H. Doha ${ }^{b}$, Taha M. Taha ${ }^{c}$ and Dumitru Baleanu ${ }^{d, e}$}

\author{
${ }^{a}$ Department of Applied Mathematics, National Research Centre \\ Dokki, 12622 Giza, Egypt \\ ${ }^{b}$ Department of Mathematics, Faculty of Science, Cairo University \\ Giza, Egypt \\ ${ }^{c}$ Department of Mathematics, Faculty of Science, Beni-Suef University \\ Beni-Suef, Egypt \\ ${ }^{d}$ Department of Mathematics, Cankaya University \\ Ankara, Turkey \\ ${ }^{e}$ Institute of Space Sciences \\ Magurele-Bucharest, Romania \\ E-mail(corresp.): ma.zaky@yahoo.com
}

Received September 2, 2017; revised February 2, 2018; accepted February 2, 2018

\begin{abstract}
To broaden the range of applicability of variable-order fractional differential models, reliable numerical approaches are needed to solve the model equation. In this paper, we develop Laguerre spectral collocation methods for solving variableorder fractional initial value problems on the half line. Specifically, we derive threeterm recurrence relations to efficiently calculate the variable-order fractional integrals and derivatives of the modified generalized Laguerre polynomials, which lead to the corresponding fractional differentiation matrices that will be used to construct the collocation methods. Comparison with other existing methods shows the superior accuracy of the proposed spectral collocation methods.
\end{abstract}

Keywords: spectral collocation methods, modified generalized Laguerre polynomials, variable order fractional integrals and derivatives, Bagley-Torvik equation.

AMS Subject Classification: 42C05; 65D99; 35R11; 65N35.

Copyright (c) 2018 The Author(s). Published by VGTU Press

This is an Open Access article distributed under the terms of the Creative Commons Attribution License (http://creativecommons.org/licenses/by/4.0/), which permits unrestricted use, distribution, and reproduction in any medium, provided the original author and source are credited. 


\section{Introduction}

The variable-order fractional (VO-F) operators [8,17], which are generalizations of constant-order fractional operators [23], open up new possibilities for robust mathematical modeling and simulation of diverse physical problems in science and engineering, such as modeling of diffusive-convective effects on the oscillatory flows [15], linear and nonlinear oscillators with viscoelastic damping [8], processing of geographical data using VO-F derivatives [9], constitutive laws in viscoelastic continuum mechanics [16], signature verification through variable/adaptive fractional order differentiators [21], anomalous diffusion problems $[10,24]$ and chloride ions sub-diffusion in concrete structures [22]. The VO-F operators can be employed to depict the variable memory of systems [12].

The VO-F operators are nonlocal with singular kernels, which makes the VO-F models complicated. Hence, the solution of VO-F models are also more complicated. Numerical computation of the VO-F operators is the key to understand the behavior and physical meaning of the VO-F models. Fu et al. [10] applied the method of approximate particular solutions to VO-F diffusion models. Cao and Qiu [7] proposed a second order numerical approximation via the VO-F weighted and shifted Grünwald-Letnikov formula to VO-F Riemann-Liouville derivative, and used it to solve VO-F ordinary differential equations. Zayernouri and Karniadakis [25] introduced fractional spectral collocation methods for linear and nonlinear VO-F differential equations. Atangana et al. [3] developed the Crank-Nicholson scheme to handle the time VO-F telegraph equation. Bhrawy et al. proposed accurate spectral collocation methods for VO-F differential equations such as Schrödinger equation [6], Galilei invariant advection diffusion equations [1], diffusion equation [24] and cable equation [5]. Moghaddam et al $[13,14]$ developed accurate and robust algorithms for approximating VO-F derivatives and integrals. Tayebi et al [20] proposed an accurate and robust meshless method based on the moving least squares approximation and the finite difference scheme for the numerical solution of VO-F advection-diffusion equation on two-dimensional arbitrary domains.

In this paper, we focus on the computation of the VO-F integrals and derivatives of the modified generalized Laguerre polynomials. Applications of the constructed computations are illustrated to compute the VO-F Caputo derivative. Besides, using the modified generalized Laguerre polynomials as the basis functions, we develop Laguerre-Gauss collocation methods to solve fractional differential equations of variable and constant orders on the half line.

This paper is organized as follows. Section 2 presents the fundamentals of VO-F operators and properties of the modified generalized Laguerre polynomials. Numerical algorithms for calculating the VO-F integral and the Caputo derivative are presented in Sections 3 and 4, respectively. The applications of the algorithms are illustrated in Section 5. Numerical examples are presented in Section 6, and the conclusion is drawn in the last section.

\section{Preliminaries and fundamentals}

In this section, we concisely point out some definitions of the VO-F operators [8, $12,17]$. We then collect some important properties of the modified generalized 
Laguerre polynomials [4]. Assume that $u(x)=0$ for $x<0$.

1. The following VO-F integral operator was proposed in [17]

$$
{ }_{0} I_{x}^{\varrho(x)}[u]:=x \mapsto \frac{1}{\Gamma(\varrho(x))} \int_{0}^{x}(x-r)^{\varrho(x)-1} u(r) d r, \quad x \geq 0 .
$$

2. In [12] several definitions were proposed. The first is identical to (2.1). The next one, is

$$
{ }_{0} I_{x}^{\varrho(x)}[u]:=x \mapsto \int_{0}^{x} \frac{(x-r)^{\varrho(r)-1}}{\Gamma(\varrho(r))} u(r) d r, \quad x \geq 0 .
$$

3. The following operator was introduced in [12]

$$
{ }_{0} I_{x}^{\varrho(x)}[u]:=x \mapsto \int_{0}^{x} \frac{(x-r)^{\varrho(x-r)-1}}{\Gamma(\varrho(x-r))} u(r) d r \quad x \geq 0 .
$$

The VO-F Caputo derivative could now be defined, as in the case of constant order [17], as follows

$$
{ }_{0}^{C} D_{x}^{\varrho(x)}:={ }_{0} I_{x}^{n-q(x, r)} \circ \frac{d^{n}}{d x^{n}},
$$

where $q(x, r)=\varrho(x), q(x, r)=\varrho(r)$ and $q(x, r)=\varrho(x-r)$, in cases $(2.1)-(2.2)$. Thus, we obtain, respectively:

1. The type I: left Caputo fractional derivative of order $\varrho(x)$

$$
{ }_{0}^{C} \mathcal{D}_{x}^{\varrho(x)} u(x)=\frac{1}{\Gamma(n-\varrho(x))} \int_{0}^{x} \frac{u^{(n)}(r) d r}{(x-r)^{\varrho(x)-n+1}} .
$$

2. The type II: left Caputo fractional derivative of order $\varrho(x)$

$$
{ }_{0}^{C} \mathfrak{D}_{x}^{\varrho(x)} u(x)=\int_{0}^{x} \frac{1}{\Gamma(n-\varrho(r))} \frac{u^{(n)}(r) d r}{(x-s) \varrho(r)-n+1} .
$$

3. The type III: left Caputo fractional derivative of order $\varrho(x)$

$$
{ }_{0}^{C} \mathbb{D}_{x}^{\varrho}{ }^{(x)} u(x)=\int_{0}^{x} \frac{1}{\Gamma(n-\varrho(x-r))} \frac{u^{(n)}(r) d r}{(x-r)^{\varrho(x-r)-n+1}},
$$

where $n-1<\varrho(\cdot)<n \in \mathbb{N}$. Such operators have been used by researchers, for examples, Coimbra et al. $[8,18]$ employed the first type in the modeling of viscous-viscoelastic oscillator. Ingman and Suzdalnitsky [11] used the second type in the modeling of viscoelastic deformation process. Atanackovic and Pilipovic [2] used the third type in generalization of Hamilton's principle. Sun et al. [19] introduced a comparative investigation of constant-order fractional derivative and the first two types of $\mathrm{VO}-\mathrm{F}$ derivatives in characterizing the memory property of systems. However, the differences between the three types 
in applications are still not clear. There are other definitions of VO-F derivatives [2]. In this paper, we will focus our attention on the first type of VO-F integrals and derivatives, i.e. $q(x, s)=\varrho(x)$.

The operator ${ }_{0}^{C} \mathcal{D}_{x}^{\varrho(x)}$ satisfies the following property $(n-1<\varrho(x) \leq n \in \mathbb{N})$

$$
{ }_{0}^{C} \mathcal{D}_{x}^{\varrho(x)} x^{\gamma}= \begin{cases}0, & \gamma=0, \ldots, n-1, \\ \frac{\Gamma(\gamma+1)}{\Gamma(\gamma+1-\varrho(x))} x^{\gamma-\varrho(x)}, & \gamma=n, n+1, \ldots .\end{cases}
$$

Next, let us introduce some properties of the modified generalized Laguerre polynomials [4]. Let $\Lambda=\{x \mid 0<x<\infty\}$ and $\chi(x)$ be a certain weight function on $\Lambda$ in the usual sense. Define

$$
L_{\chi}^{2}(\Lambda)=\left\{y \mid y \text { is measurable \& }\|y\|_{\chi}<\infty\right\},
$$

which is a Hilbert space, equipped with the following inner product and norm:

$$
(u, y)_{\chi}=\int_{\Lambda} \chi(x) u(x) y(x) d x, \quad\|y\|_{\chi}=(y, y)_{\chi}^{\frac{1}{2}} .
$$

In principle, the generalized Laguerre polynomials are suitable for the approximation of functions with growth at infinity. We now recall some properties of the generalized Laguerre polynomials $\mathcal{L}_{i}^{(\theta, \beta)}(x)$.

Let $\chi^{(\theta, \beta)}=x^{\theta} e^{-\beta x}, \theta>-1$, and $\beta>0, \partial_{x} v(x)=\frac{\partial}{\partial x} v(x)$. The corresponding generalized Laguerre polynomials of degree $i$ are defined by

$$
\mathcal{L}_{i}^{(\theta, \beta)}(x)=\frac{1}{i !} x^{-\theta} e^{\beta x} \partial_{x}^{i}\left(x^{i+\theta} e^{-\beta x}\right), \quad i=0,1,2, \ldots
$$

They are the eigenfunctions of the Sturm-Liouville problem

$$
\partial_{x}\left(\chi^{(\theta+1, \beta)}(x) \partial_{x} v(x)\right)+\lambda_{i}^{(\beta)} \chi^{(\theta, \beta)}(x) v(x)=0, \quad 0<x<\infty
$$

with the corresponding eigenvalues $\lambda_{i}^{(\beta)}=i \beta$. They fulfill the following threeterm recurrence relations:

$$
\begin{aligned}
& \mathcal{L}_{0}^{(\theta, \beta)}(x)=1, \quad \mathcal{L}_{1}^{(\theta, \beta)}(x)=-\beta x+\frac{\Gamma(\theta+2)}{\Gamma(\theta+1)}, \\
& \mathcal{L}_{i+1}^{(\theta, \beta)}(x)=\frac{2 i+\theta+1-\beta x}{i+1} \mathcal{L}_{i}^{(\theta, \beta)}(x)-\frac{i+\theta}{i+1} \mathcal{L}_{i-1}^{(\theta, \beta)}(x), \quad i \geq 1
\end{aligned}
$$

and

$$
\mathcal{L}_{i}^{(\theta, \beta)}(x)=\mathcal{L}_{i}^{(\theta+1, \beta)}(x)-\mathcal{L}_{i-1}^{(\theta+1, \beta)}(x)=\frac{1}{\beta}\left(\partial_{x} \mathcal{L}_{i}^{(\theta, \beta)}(x)-\partial_{x} \mathcal{L}_{i+1}^{(\theta, \beta)}(x)\right) .
$$

The $m$-th derivative of a generalized Laguerre polynomial satisfies the relation

$$
\partial_{x}^{m} \mathcal{L}_{i}^{(\theta, \beta)}(x)=(-\beta)^{m} \mathcal{L}_{i-m}^{(\theta+m, \beta)}(x) .
$$

The generalized Laguerre polynomials form a complete $L_{\chi^{\theta, \beta}}^{2}(\Lambda)$-orthogonal system,

$$
\left(\mathcal{L}_{i}^{(\theta, \beta)}(x), \mathcal{L}_{j}^{(\theta, \beta)}(x)\right)_{\chi^{(\theta, \beta)}}=\gamma_{i}^{(\theta, \beta)} \delta_{i, j}, \quad \gamma_{i}^{(\theta, \beta)}=\frac{\Gamma(i+\theta+1)}{\beta^{\theta+1} \Gamma(i+1)},
$$

where $\delta_{i, j}$ is the Kronecker symbol. 


\section{Approximation to the variable-order fractional integral}

The main goal of this section is to develop a new algorithm to approximate the $\mathrm{VO}-\mathrm{F}$ integral of a given function.

Let $u \in L_{\chi^{\theta, \beta}}^{2}(\Lambda)$ and $N$ be a positive integer, then, we can expand it in terms of generalized Laguerre polynomials as

$$
u(x) \simeq u_{N}(x)=\sum_{i=0}^{N} \ell_{i}^{(\theta, \beta)} \mathcal{L}_{i}^{(\theta, \beta)}(x) .
$$

If $u_{N}(x)$ is an orthogonal projection of $u(x)$, then $\ell_{i}^{(\theta, \beta)}$ can be determined by the orthogonality of $\left\{\mathcal{L}_{i}^{(\theta, \beta)}(x)\right\}$. If $u_{N}(x)$ is the interpolation of $u(x)$ on the generalized Laguerre-Gauss points $\left\{x_{N, i}^{\theta, \beta}\right\}_{i=0}^{N}$, then $\ell_{i}^{(\theta, \beta)}$ can be determined by

$$
\ell_{i}^{(\theta, \beta)}=\frac{1}{\gamma_{i}^{(\theta, \beta)}} \sum_{j=0}^{N} u\left(x_{N, j}^{(\theta, \beta)}\right) \mathcal{L}_{i}^{(\theta, \beta)}\left(x_{N, j}^{(\theta, \beta)}\right) \chi_{N, j}^{(\theta, \beta)}
$$

where $\chi_{N, j}^{(\theta, \beta)}$ are the corresponding weights. In this paper, we assume that $u_{N}(x)$ is the interpolation of $u(x)$.

Therefore, for any $n-1<\varrho_{\min }<\varrho(x)<\varrho_{\max }<n$, the VO-F integral ${ }_{0} I_{x}^{\varrho(x)} u(x)$ can be approximated by

$$
\begin{gathered}
{ }_{0} I_{x}^{\varrho(x)} u(x) \approx{ }_{0} I_{x}^{\varrho(x)} u_{N}(x)=\frac{1}{\Gamma(\varrho(x))} \int_{0}^{x}(x-t)^{\varrho(x)-1} u_{N}(t) d t=\frac{1}{\Gamma(\varrho(x))} \\
\times \sum_{i=0}^{N} \ell_{i}^{(\theta, \beta)} \int_{0}^{x}(x-t)^{\varrho(x)-1} \mathcal{L}_{i}^{(\theta, \beta)}(t) d t=\sum_{i=0}^{N} \ell_{i}^{(\theta, \beta)} \hat{\mathcal{L}}_{i}^{(\varrho(x), \theta, \beta)}(x),
\end{gathered}
$$

where

$$
\hat{\mathcal{L}}_{i}^{(\varrho(x), \theta, \beta)}(x)=\frac{1}{\Gamma(\varrho(x))} \int_{0}^{x}(x-t)^{\varrho(x)-1} \mathcal{L}_{i}^{(\theta, \beta)}(t) d t .
$$

Next, we develop a recurrence formula to calculate the $\varrho(x)$ th-order fractional integral of $\mathcal{L}_{i}^{(\theta, \beta)}$. From (2.4), we can easily get

$$
\hat{\mathcal{L}}_{0}^{(\varrho(x), \theta, \beta)}(x)=\frac{x^{\varrho(x)}}{\Gamma(\varrho(x)+1)}, \quad \hat{\mathcal{L}}_{1}^{(\varrho(x), \theta, \beta)}(x)=\frac{x^{\varrho(x)}}{\Gamma(\varrho(x)+1)}-\frac{\beta x^{\varrho(x)+1}}{\Gamma(\varrho(x)+2)} .
$$

For $i \geq 1$, we have

$$
\begin{aligned}
& \hat{\mathcal{L}}_{i+1}^{(\varrho(x), \theta, \beta)}(x)=\frac{1}{\Gamma(\varrho(x))} \int_{0}^{x}(x-t)^{\varrho(x)-1} \mathcal{L}_{i+1}^{(\theta, \beta)}(t) d t=\frac{1}{\Gamma(\varrho(x))} \frac{1}{i+1} \\
& \quad \times \int_{0}^{x}(x-t)^{\varrho(x)-1} \times \quad\left[(2 i+\theta+1-\beta t) \mathcal{L}_{i}^{(\theta, \beta)}(t)-(i+\theta) \mathcal{L}_{i-1}^{(\theta, \beta)}(t)\right] d t \\
& =\frac{1}{i+1}\left\{(2 i+\theta+1-\beta x) \hat{\mathcal{L}}_{i}^{(\varrho(x), \theta, \beta)}(x)-(i+\theta) \hat{\mathcal{L}}_{i-1}^{(\varrho(x), \theta, \beta)}(x)\right. \\
& \left.\quad+\frac{\beta}{\Gamma(\varrho(x))} \int_{0}^{x}(x-t)^{\varrho(x)} \mathcal{L}_{i}^{(\theta, \beta)}(t) d t\right\} .
\end{aligned}
$$


We can verify from formula (2.5) that for $i \geq 1$,

$$
\begin{aligned}
\hat{\mathcal{L}}_{i+1}^{(\varrho(x), \theta, \beta)}(x)= & \frac{1}{i+1}\left\{(2 i+\theta+1-\beta x) \hat{\mathcal{L}}_{i}^{(\varrho(x), \theta, \beta)}(x)-(i+\theta) \hat{\mathcal{L}}_{i-1}^{(\varrho(x), \theta, \beta)}(x)\right. \\
& \left.+\frac{1}{\Gamma(\varrho(x))} \int_{0}^{x}(x-t)^{\varrho(x)}\left(\partial_{t} \mathcal{L}_{i}^{(\theta, \beta)}(t)-\partial_{t} \mathcal{L}_{i+1}^{(\theta, \beta)}(t)\right) d t\right\} \\
= & \frac{1}{i+1}\left\{(2 i+\theta+1-\beta x) \hat{\mathcal{L}}_{i}^{(\varrho(x), \theta, \beta)}(x)-(i+\theta) \hat{\mathcal{L}}_{i-1}^{(\varrho(x), \theta, \beta)}(x)\right. \\
& +\frac{1}{\Gamma(\varrho(x))}\left[(x-t)^{\varrho(x)}\left(\mathcal{L}_{i}^{(\theta, \beta)}(t)-\mathcal{L}_{i+1}^{(\theta, \beta)}(t)\right)\right]_{0}^{x} \\
& \left.+\varrho(x)\left(\hat{\mathcal{L}}_{i}^{(\varrho(x), \theta, \beta)}(x)-\hat{\mathcal{L}}_{i+1}^{(\varrho(x), \theta, \beta)}(x)\right)\right\} \\
= & \frac{1}{(i+\varrho(x)+1)}\left\{(2 i+\theta+\varrho(x)+1-\beta x) \hat{\mathcal{L}}_{i}^{(\varrho(x), \theta, \beta)}(x)\right. \\
& \left.-(i+\theta) \hat{\mathcal{L}}_{i-1}^{(\varrho(x), \theta, \beta)}(x)-\frac{x^{\varrho(x)}}{\Gamma(\varrho(x))}\left(\mathcal{L}_{i}^{(\theta, \beta)}(0)-\mathcal{L}_{i+1}^{(\theta, \beta)}(0)\right)\right\} .
\end{aligned}
$$

Hence, for $i \geq 1$, we get the following recurrence relation

$$
\begin{aligned}
\hat{\mathcal{L}}_{i+1}^{(\varrho(x), \theta, \beta)}(x)= & \frac{1}{(i+\varrho(x)+1)}\left\{(2 i+\theta+\varrho(x)+1-\beta x) \hat{\mathcal{L}}_{i}^{(\varrho(x), \theta, \beta)}(x)\right. \\
& \left.-(i+\theta) \hat{\mathcal{L}}_{i-1}^{(\varrho(x), \theta, \beta)}(x)-\frac{x^{\varrho(x)}}{\Gamma(\varrho(x))}\left(\mathcal{L}_{i}^{(\theta, \beta)}(0)-\mathcal{L}_{i+1}^{(\theta, \beta)}(0)\right)\right\} .
\end{aligned}
$$

So, $\hat{\mathcal{L}}_{i}^{(\varrho(x), \theta, \beta)}(x)$ can be calculated by the following formula

$$
\begin{aligned}
& \hat{\mathcal{L}}_{0}^{(\varrho(x), \theta, \beta)}(x)=\frac{x^{\varrho(x)}}{\Gamma(\varrho(x)+1)}, \quad \hat{\mathcal{L}}_{1}^{(\varrho(x), \theta, \beta)}(x)=\frac{x^{\varrho(x)}}{\Gamma(\varrho(x)+1)}-\frac{\beta x^{\varrho(x)+1}}{\Gamma(\varrho(x)+2)} \\
& \hat{\mathcal{L}}_{i+1}^{(\varrho(x), \theta, \beta)}(x)=\frac{1}{(i+\varrho(x)+1)}\left\{(2 i+\theta+\varrho(x)+1-\beta x) \hat{\mathcal{L}}_{i}^{(\varrho(x), \theta, \beta)}(x)\right. \\
& \left.\quad-(i+\theta) \hat{\mathcal{L}}_{i-1}^{(\varrho(x), \theta, \beta)}(x)-\frac{x^{\varrho(x)}}{\Gamma(\varrho(x))}\left(\mathcal{L}_{i}^{(\theta, \beta)}(0)-\mathcal{L}_{i+1}^{(\theta, \beta)}(0)\right)\right\}, \quad i \geq 1
\end{aligned}
$$

Therefore, ${ }_{0} I_{x}^{\varrho(x)} u(x)$ can be approximated by

$$
{ }_{0} I_{x}^{\varrho(x)} u(x) \approx{ }_{0} I_{x}^{\varrho(x)} u_{N}(x)=\sum_{i=0}^{N} \ell_{i}^{(\theta, \beta)} \hat{\mathcal{L}}_{i}^{(\varrho(x), \theta, \beta)}(x),
$$

where $\hat{\mathcal{L}}_{i}^{(\varrho(x), \theta, \beta)}(x)$ is given by $(3.2)$, and $\ell_{i}^{(\theta, \beta)}$ is given by (3.1).

Remark 1. When $\varrho(x)=\varrho=$ constant, then the operator of VO-F integral is reduced to corresponding integral of constant order and the approximation relation (3.3) reduces to

$$
{ }_{0} I_{x}^{\varrho} u(x) \approx{ }_{0} I_{x}^{\varrho} u_{N}(x)=\sum_{i=0}^{N} \ell_{i}^{(\theta, \beta)} \hat{\mathcal{L}}_{i}^{(\varrho, \theta, \beta)}(x) .
$$




\section{Approximation to the variable-order fractional Caputo derivative}

In this section, we describe how to use the generalized Laguerre polynomials to numerically approximate the VO-F derivative in the Caputo sense of a given function $u(x), x \in \Lambda$. The computerized mathematical algorithm is based on the numerical approximation of the VO-F integral derived in the previous section.

Suppose that $u_{N}(x)$ is the approximate polynomial of $u(x)$, which can be expressed by

$$
u_{N}(x)=\sum_{i=0}^{N} \ell_{i}^{(\theta, \beta)} \mathcal{L}_{i}^{(\theta, \beta)}(x), \quad x \in \Lambda .
$$

Let $n-1<\varrho_{\min }<\varrho(x)<\varrho_{\max }<n \in \mathbb{N}$, we approximate the VO-F derivative ${ }_{0}^{C} \mathcal{D}_{x}^{\varrho(x)} u(x)$ by the generalized Laguerre polynomials as

$$
\begin{aligned}
{ }_{0}^{C} & \mathcal{D}_{x}^{\varrho(x)} u_{N}(x)=\frac{1}{\Gamma(n-\varrho(x))} \int_{0}^{x}(x-t)^{n-\varrho(x)-1} \partial_{t}^{n} u_{N}(t) d t \\
& =\frac{1}{\Gamma(n-\varrho(x))} \sum_{i=0}^{N} \ell_{i}^{(\theta, \beta)} \int_{0}^{x}(x-t)^{n-\varrho(x)-1} \partial_{t}^{n} \mathcal{L}_{i}^{(\theta, \beta)}(t) d t \\
& =\sum_{i=0}^{N} \ell_{i}^{(\theta, \beta)}(-\beta)^{n}\left(\frac{1}{\Gamma(n-\varrho(x))} \int_{0}^{x}(x-t)^{n-\varrho(x)-1} \mathcal{L}_{i-n}^{(\theta+n, \beta)}(t) d t\right) \\
& =\sum_{i=0}^{N} \ell_{i}^{(\theta, \beta)}(-\beta)^{n} \hat{\mathcal{L}}_{i-n}^{(n-\varrho(x), \theta+n, \beta)}(x),
\end{aligned}
$$

where $\hat{\mathcal{L}}_{i-n}^{(n-\varrho(x), \theta+n, \beta)}(x)=0$ for $0 \leq i \leq n-1$.

Therefore, the VO-F derivative of $u(x)$ can be approximated by

$$
{ }_{0}^{C} \mathcal{D}_{x}^{\varrho(x)} u_{N}(x)=\sum_{i=0}^{N} \ell_{i}^{(\theta, \beta)}(-\beta)^{n} \hat{\mathcal{L}}_{i-n}^{(n-\varrho(x), \theta+n, \beta)}(x)=\sum_{i=0}^{N} \ell_{i}^{(\theta, \beta)} D_{i, n, \theta, \beta}^{(\varrho(x))}(x),
$$

which alternatively may be written in the matrix form:

$$
{ }_{0}^{C} \mathcal{D}_{x}^{\varrho(x)} u_{N}(x)=\mathcal{L}^{(\theta, \beta)} \mathbf{D}_{n, \theta, \beta}^{(\varrho(x))}(x)
$$

with $\mathcal{L}^{(\theta, \beta)}=\left[\ell_{0}^{(\theta, \beta)}, \ell_{1}^{(\theta, \beta)}, \ldots, \ell_{N}^{(\theta, \beta)}\right]$,

$$
\mathbf{D}_{n, \theta, \beta}^{(\varrho(x))}(x)=\left[D_{0, n, \theta, \beta}^{(\varrho(x))}(x), D_{1, n, \theta, \beta}^{(\varrho(x))}(x), \ldots, D_{N, n, \theta, \beta}^{(\varrho(x))}(x)\right]^{T},
$$

where $n-1<\varrho_{\min }<\varrho(x)<\varrho_{\max }<n \in \mathbb{N}$ and

$$
\begin{aligned}
D_{i, n, \theta, \beta}^{(\varrho(x))}(x) & =\frac{1}{\Gamma(n-\varrho(x))} \int_{0}^{x}(x-t)^{n-\varrho(x)-1} \partial_{t}^{n} \mathcal{L}_{i}^{(\theta, \beta)}(t) d t \\
& =(-\beta)^{n} \hat{\mathcal{L}}_{i-n}^{(n-\varrho(x), \theta+n, \beta)}, \quad i \geq n
\end{aligned}
$$

and $D_{i, n, \theta, \beta}^{(\varrho(x))}(x)=0$ for $0 \leq i \leq n-1$. 


\section{Applications of the algorithms}

After the construction of the VO-F differentiation matrix of Caputo type (4.1), we now use the generalized Laguerre spectral collocation method together with the generalized Laguerre operational matrix of VO-F derivative to solve the following VO-F differential equation:

$$
\begin{aligned}
& a(x) u^{\prime}(x)+b(x)_{0}^{C} \mathcal{D}_{x}^{\varrho(x)} u(x)+c(x) u(x)=f(x), \quad x \in \Lambda, \\
& u(0)=u_{0}
\end{aligned}
$$

where $0<\varrho_{\min }<\varrho(x)<\varrho_{\max }<1, a(x), b(x), c(x)$ and $f(x)$ are real-valued functions. A special case of (5.1) occurs when $\varrho(x)=\varrho=\frac{1}{2}$, corresponding to the fractional Basset equation. This model represents a classical problem in fluid dynamics in the scope of an unsteady motion of a particle that accelerates in a viscous fluid under the action of gravity.

Suppose that $u_{N}(x)$ is the approximate solution of $u(x)$ and $x_{i}(0 \leq i \leq N)$ is the generalized Laguerre-Gauss nodes of $\mathcal{L}_{N+1}^{(\theta, \beta)}(x)$. Now, using (4.1) then it is easy to write

$$
\begin{aligned}
& a\left(x_{i}\right) \mathcal{L}^{(\theta, \beta)} \mathbf{D}_{1, \theta, \beta}^{(1)}\left(x_{i}\right)+b\left(x_{i}\right) \mathcal{L}^{(\theta, \beta)} \mathbf{D}_{1, \theta, \beta}^{\left(\varrho\left(x_{i}\right)\right)}\left(x_{i}\right)+c\left(x_{i}\right) \mathcal{L}^{(\theta, \beta)} \mathbf{D}_{0, \theta, \beta}^{(0)}\left(x_{i}\right)=f\left(x_{i}\right), \\
& u_{N}(0)=u_{0}, \quad i=0,1, \ldots, N-1 .
\end{aligned}
$$

Let us denote $F=\left[f\left(x_{0}\right), \ldots, f\left(x_{N-1}\right), u_{0}\right]$. Then $(5.2)$ is equivalent to the following matrix equation

$$
\mathcal{L}^{(\theta, \beta)} E=F,
$$

where

$$
E=\left(e_{i j}\right)=\left\{\begin{array}{c}
a\left(x_{j}\right) D_{i, 1, \theta, \beta}^{(1)}\left(x_{j}\right)+b\left(x_{j}\right) D_{i, 1, \theta, \beta}^{\left(\varrho\left(x_{j}\right)\right)}\left(x_{j}\right),+c\left(x_{j}\right) D_{i, 0, \theta, \beta}^{(0)}\left(x_{j}\right), \\
0 \leq i \leq N, 0 \leq j \leq N-1, \\
\frac{\Gamma(i+\theta+1)}{\Gamma(1+\theta) i !}, \quad 0 \leq i \leq N, j=N .
\end{array}\right.
$$

For the following fractional initial value problem

$$
\begin{aligned}
& a(x) u^{(m)}(x)+b(x)_{0}^{C} \mathcal{D}_{x}^{\varrho(x)} u(x)+c(x) u(x)=f(x), \quad x \in \Lambda, \\
& u(0)=u_{0}, \quad u^{\prime}(0)=v_{0},
\end{aligned}
$$

where $1<\varrho_{\min }<\varrho(x)<\varrho_{\max }<2, m=1$ or 2 , we can also get the algebraic equation of the form like $(5.2)$, where $F=\left[f\left(x_{0}\right), \ldots, f\left(x_{N-2}\right), u_{0}, v_{0}\right]$ and

$$
E=\left(e_{i j}\right)=\left\{\begin{array}{l}
a\left(x_{j}\right) D_{i, m, \theta, \beta}^{(m)}\left(x_{j}\right)+b\left(x_{j}\right) D_{i, 2, \theta, \beta}^{\left(\varrho\left(x_{j}\right)\right)}\left(x_{j}\right),+c\left(x_{j}\right) D_{i, 0, \theta, \beta}^{(0)}\left(x_{j}\right), \\
0 \leq i \leq N, \quad 0 \leq j \leq N-2, \\
\frac{\Gamma(i+\theta+1)}{\Gamma(1+\theta) i !}, \quad 0 \leq i \leq N, j=N-1, \\
D_{i, 1, \theta, \beta}^{(1)}(0), \quad 0 \leq i \leq N, j=N .
\end{array}\right.
$$


In our implementation, these systems have been solved using the Mathematica function FindRoot with zero initial approximation.

A special case of (5.3) occurs when $m=2$ and $\varrho(x)=\varrho=\frac{3}{2}$, corresponding to the fractional Bagley-Torvik equation. The fractional Bagley-Torvik equation is important for modeling the motion of a thin rigid plate immersed in a Newtonian fluid.

\section{$6 \quad$ Numerical examples}

In this section we present three numerical examples to illustrate the accuracy and efficiency of the algorithms presented in the previous sections. The first one is introduced to test the accuracy of the formula (4.1).

Example 1. Let $u(x)=e^{x}, x \in[0,1]$. Now we numerically calculate the VO-F derivative ${ }_{0}^{C} \mathcal{D}_{x}^{\varrho(x)} u(x), n-1 \leq \varrho(x) \leq n$.

The analytical form of the VO-F derivative of $u(x)$ is given by

$$
{ }_{0}^{C} \mathcal{D}_{x}^{\varrho(x)} e^{x}=e^{x}\left(1-\frac{\Gamma(n-\varrho(x), x)}{\Gamma(n-\varrho(x))}\right) .
$$

In Tables 1 and 2, we list the maximum absolute errors (AEs) between the exact solution $u(x)$ and the approximate solutions $u_{N}$ at different constantand variable-orders, respectively.

Table 1. The maximum AEs at different constant fractional orders for Example 1.

\begin{tabular}{llllllll}
\hline$N$ & $(\theta, \beta)$ & $\varrho=0.2$ & $\varrho=0.5$ & $\varrho=0.8$ & $\varrho=1.2$ & $\varrho=1.5$ & $\varrho=1.8$ \\
\hline 10 & $(1,3)$ & $7.93-3$ & $1.46-2$ & $3.36-2$ & $1.10-1$ & $1.78-1$ & $3.62-1$ \\
20 & & $1.53-5$ & $3.45-5$ & $9.72-5$ & $4.10-4$ & $8.24-4$ & $2.07-3$ \\
40 & & $3.07-11$ & $8.49-11$ & $2.93-10$ & $1.61-9$ & $4.00-9$ & $1.25-8$ \\
80 & & $4.88-15$ & $6.21-15$ & $5.77-15$ & $7.32-15$ & $1.55-14$ & $1.37-14$ \\
\hline 10 & $(2,6)$ & $2.93-6$ & $6.04-6$ & $1.55-5$ & $5.76-5$ & $1.06-4$ & $2.48-4$ \\
20 & & $1.09-12$ & $2.73-12$ & $8.64-12$ & $4.13-11$ & $9.46-11$ & $2.73-10$ \\
40 & & $1.33-15$ & $2.66-15$ & $2.67-15$ & $1.77-15$ & $3.10-15$ & $2.66-15$ \\
80 & & $1.33-15$ & $2.66-15$ & $2.67-15$ & $1.77-15$ & $3.10-15$ & $2.66-15$ \\
\hline
\end{tabular}

Example 2. Consider the following VO-F Bagley-Torvik equation

$$
\begin{aligned}
& u^{\prime \prime}(x)+{ }_{0}^{C} \mathcal{D}_{x}^{\varrho(x)} u(x)+u(x)=f(x), \quad x \in(0, L], \\
& u(0)=0, \quad u^{\prime}(0)=1 .
\end{aligned}
$$

The exact solution is $u(x)=\sin x$.

Table 3 displays the maximum AEs of the proposed method at $\varrho(x)=\frac{3}{2}$ and $\varrho(x)=\frac{9+\sin (x-10)}{5}$ for different chooses of $\theta, \beta$ and $N$. Figure 1 shows the logarithmic graphs of the AEs at $\varrho(x)=\frac{9+\sin (x-10)}{5}, N=25$ and different chooses of $\theta=\beta$. 
Table 2. The maximum AEs at different variable orders for Example 1.

\begin{tabular}{llll}
\hline$(\theta, \beta)$ & $N$ & $\varrho(x)=\frac{9+\sin t}{10}$ & $\varrho(x)=\frac{3+\tanh t}{2}$ \\
\hline$(2,4)$ & 10 & $4.648 \times 10^{-3}$ & $1.833 \times 10^{-2}$ \\
& 20 & $4.556 \times 10^{-7}$ & $2.598 \times 10^{-6}$ \\
& 30 & $2.282 \times 10^{-11}$ & $1.625 \times 10^{-10}$ \\
& 40 & $5.329 \times 10^{-15}$ & $7.688 \times 10^{-15}$ \\
\hline$(3,6)$ & 10 & $9.862 \times 10^{-5}$ & $4.228 \times 10^{-4}$ \\
& 20 & $1.013 \times 10^{-10}$ & $6.287 \times 10^{-10}$ \\
& 30 & $3.997 \times 10^{-15}$ & $3.552 \times 10^{-15}$ \\
& 40 & $3.997 \times 10^{-15}$ & $3.552 \times 10^{-15}$ \\
\hline
\end{tabular}

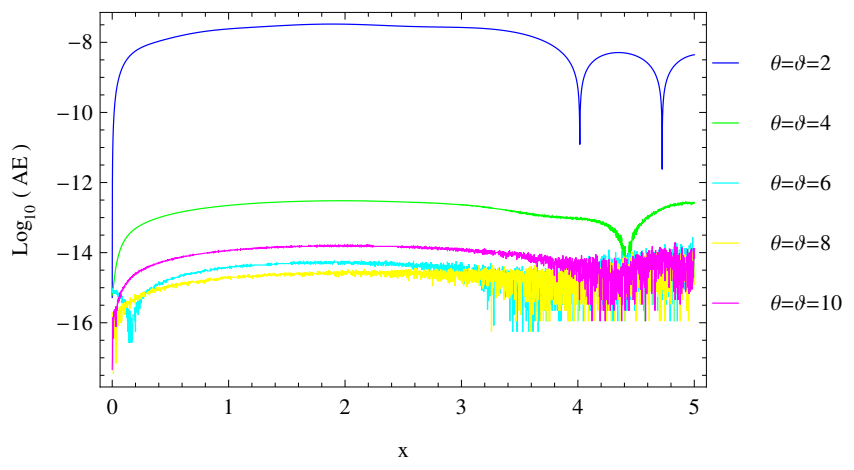

Figure 1. Logarithmic graphs of the $\mathrm{AE}$ at $\varrho(x)=\frac{9+\sin (x-10)}{5}, N=25$ and various choices of $\theta=\beta$ for Example 2 .

Example 3. Consider the following VO-F Bagley-Torvik equation

$$
\begin{aligned}
& u^{\prime \prime}(x)+{ }_{0}^{C} \mathcal{D}_{x}^{\varrho(x)} u(x)+u(x)=\frac{\Gamma(4)}{\Gamma(4-\varrho(x))} x^{3-\varrho(x)}+x^{3}+7 x+1, x \in\left(0, \frac{\pi}{2}\right], \\
& u(0)=u^{\prime}(0)=1 .
\end{aligned}
$$

The exact solution is $u(x)=x^{3}+x+1$.

In this example, we consider two cases, $\varrho(x)=1.5$ and $\varrho(x)=1+0.5|\sin x|$, $x \in\left[0, \frac{\pi}{2}\right]$. Table 4 displays a comparison between the M- and IM-algorithms $[13,14]$ by means of the maximum AEs.

\section{Conclusions}

In this paper, an efficient three-term recurrence relation to calculate both VO$\mathrm{F}$ integrals and derivatives of the modified generalized Laguerre polynomials was developed. Spectral collocation methods were developed to solve VO-F differential equations. The results of this paper expand the application of 
Table 3. The maximum AEs at $L=1$ for Example 2.

\begin{tabular}{lllll}
\hline$\varrho(x)$ & $N$ & $\theta=0, \beta=1$ & $\theta=2, \beta=4$ & $\theta=3, \beta=6$ \\
\hline$\frac{3}{2}$ & 5 & $5.546 \times 10^{-3}$ & $2.916 \times 10^{-4}$ & $1.427 \times 10^{-4}$ \\
& 10 & $4.485 \times 10^{-4}$ & $1.431 \times 10^{-7}$ & $9.038 \times 10^{-9}$ \\
& 15 & $8.845 \times 10^{-6}$ & $3.675 \times 10^{-11}$ & $9.313 \times 10^{-12}$ \\
& 20 & $8.133 \times 10^{-6}$ & $2.166 \times 10^{-13}$ & $2.220 \times 10^{-15}$ \\
\hline$\frac{9+\sin (x-10)}{5}$ & 5 & $8.318 \times 10^{-3}$ & $2.666 \times 10^{-3}$ & $1.231 \times 10^{-3}$ \\
& 10 & $2.515 \times 10^{-3}$ & $3.854 \times 10^{-6}$ & $1.179 \times 10^{-7}$ \\
& 15 & $1.771 \times 10^{-4}$ & $2.721 \times 10^{-9}$ & $7.242 \times 10^{-11}$ \\
& 20 & $5.418 \times 10^{-6}$ & $1.0522 \times 10^{-12}$ & $2.742 \times 10^{-14}$ \\
\hline
\end{tabular}

Table 4. Comparison of the maximum AEs for Example 3 with $\theta=\beta=10$ and two cases of $\varrho(x), x \in\left[0, \frac{\pi}{2}\right]$.

\begin{tabular}{l|lll|ll}
\hline$\varrho(x)$ & & M-algorithm & IM-algorithm & \multicolumn{2}{c}{ Collocation method } \\
\hline \multirow{3}{*}{1.5} & $h=0.02$ & $8.16 \times 10^{-3}$ & $1.15 \times 10^{-3}$ & $N=3$ & $5.77 \times 10^{-15}$ \\
& $h=0.01$ & $4.27 \times 10^{-3}$ & $4.29 \times 10^{-4}$ & $N=4$ & $4.57 \times 10^{-15}$ \\
& $h=0.005$ & $2.18 \times 10^{-3}$ & $1.56 \times 10^{-4}$ & $N=5$ & $4.44 \times 10^{-15}$ \\
\hline \multirow{3}{*}{$1+0.5|\sin x|$} & $h=0.02$ & $7.22 \times 10^{-3}$ & $1.53 \times 10^{-4}$ & $N=3$ & $4.88 \times 10^{-15}$ \\
& $h=0.01$ & $3.70 \times 10^{-3}$ & $5.57 \times 10^{-5}$ & $N=4$ & $3.10 \times 10^{-15}$ \\
& $h=0.005$ & $1.86 \times 10^{-3}$ & $1.93 \times 10^{-5}$ & $N=5$ & $2.77 \times 10^{-15}$ \\
\hline
\end{tabular}

the Laguerre-Gauss collocation methods to VO-F problems. The suggested algorithms can be used also for solving VO-F fractional partial differential equations. Hence, the method is promising for VO-F differential equations. However, the analysis of the scheme is a challenging problem deserving further study.

\section{References}

[1] M.A. Abd-Elkawy and R.T. Alqahtani. Space-time spectral collocation algorithm for the variable-order Galilei invariant advection diffusion equations with a nonlinear source term. Mathematical Modelling and Analysis, 22(1):1-20, 2017. https://doi.org/10.3846/13926292.2017.1258014.

[2] T. Atanackovic and S. Pilipovic. Hamilton's principle with variable order fractional derivatives. Fractional Calculus and Applied Analysis, 14(1):94-109, 2011. https://doi.org/10.2478/s13540-011-0007-7.

[3] A. Atangana. On the stability and convergence of the time-fractional variable order telegraph equation. Journal of Computational Physics, 293:104-114, 2015. https://doi.org/10.1016/j.jcp.2014.12.043.

[4] D. Baleanu, A.H. Bhrawy and T.M. Taha. A modified generalized Laguerre spectral method for fractional differential equations on the half line. Abstract and Applied Analysis, 2013:1-12, 2013. https://doi.org/10.1155/2013/413529.

[5] A.H. Bhrawy and M.A. Zaky. Numerical simulation for two-dimensional variable- 
order fractional nonlinear cable equation. Nonlinear Dynamics, 80(1):101-116, 2015. https://doi.org/10.1007/s11071-014-1854-7.

[6] A.H. Bhrawy and M.A. Zaky. An improved collocation method for multi-dimensional space-time variable-order fractional Schrödinger equations. Applied Numerical Mathematics, 111:197-218, 2017. https://doi.org/10.1016/j.apnum.2016.09.009.

[7] J. Cao and Y. Qiu. A high order numerical scheme for variable order fractional ordinary differential equation. Applied Mathematics Letters, 61:88-94, 2016. https://doi.org/10.1016/j.aml.2016.05.012.

[8] C.F.M. Coimbra. Mechanics with variable-order differential operators. Annalen der Physik, 12(11-12):692-703, 2003. https://doi.org/10.1002/andp.200310032.

[9] G.R.J Cooper and D.R. Cowan. Filtering using variable order vertical derivatives. Computers \& Geosciences, 30(5):455-459, 2004. https://doi.org/10.1016/j.cageo.2004.03.001.

[10] Z.-J. Fu, W. Chen and L. Ling. Method of approximate particular solutions for constant- and variable-order fractional diffusion models. Engineering Analysis with Boundary Elements, 57:37-46, 2015. https://doi.org/10.1016/j.enganabound.2014.09.003.

[11] D. Ingman and J. Suzdalnitsky. Application of differential operator with servoorder function in model of viscoelastic deformation process. Journal of Engineering Mechanics, 131(7):763-767, 2005. https://doi.org/10.1061/(ASCE)07339399(2005)131:7(763).

[12] C.F. Lorenzo and T.T. Hartley. Variable order and distributed order fractional operators. Nonlinear Dynamics, 29(1):57-98, 2002. https://doi.org/10.1023/A:1016586905654.

[13] B.P. Moghaddam and J.A.T. Machado. Extended algorithms for approximating variable order fractional derivatives with applications. Journal of Scientific Computing, 71(3):1351-1374, 2017. https://doi.org/10.1007/s10915-016-0343-1.

[14] B.P. Moghaddam, J.A.T. Machado and H. Behforooz. An integro quadratic spline approach for a class of variable-order fractional initial value problems. Chaos, Solitons \& Fractals, 102:354-360, 2017. https://doi.org/10.1016/j.chaos.2017.03.065.

[15] H. Pedro, M. Kobayashi, J. Pereira and C. Coimbra. Variable order modeling of diffusive-convective effects on the oscillatory flow past a sphere. Journal of Vibration and Control, 14(9-10):1659-1672, 2008. https://doi.org/10.1177/1077546307087397.

[16] L. Ramirez and C.F.M. Coimbra. A variable order constitutive relation for viscoelasticity. Annalen der Physik, 16(7-8):543-552, 2007. https://doi.org/10.1002/andp.200710246.

[17] S. Samko and B. Ross. Integration and differentiation to a variable fractional orde. Integral Transforms and Special Functions, 1(4):277-300, 1993. https://doi.org/10.1080/10652469308819027.

[18] C.M. Soon, C.F.M. Coimbra and M. Kobayashi. The variable viscoelasticity oscillator. Annalen der Physik, 14(6):378-389, 2005. https://doi.org/10.1002/andp.200410140.

[19] H.G. Sun., W. Chen, H. Wei and Y.Q. Chen. A comparative study of constantorder and variable-order fractional models in characterizing memory property of 
systems. The European Physical Journal Special Topics, 193(1):185-192, 2011. https://doi.org/10.1140/epjst/e2011-01390-6.

[20] A. Tayebi, Y. Shekari and M.H. Heydari. A meshless method for solving two-dimensional variable-order time fractional advection-diffusion equation. Journal of Computational Physics, 340:655-669, 2017. https://doi.org/10.1016/j.jcp.2017.03.061.

[21] Ch.-Ch. Tseng. Design of variable and adaptive fractional order FIR differentiators. Signal Processing, 86(10):2554-2566, 2006. https://doi.org/10.1016/j.sigpro.2006.02.004.

[22] S. Wei, W. Chen and J. Zhang. Time-fractional derivative model for chloride ions sub-diffusion in reinforced concrete. European Journal of Environmental and Civil Engineering, 21(3):319-331, 2017. https://doi.org/10.1080/19648189.2015.1116467.

[23] M.A. Zaky. A Legendre spectral quadrature tau method for the multi-term time-fractional diffusion equations. Computational and Applied Mathematics, pp. 1-14, 2017. https://doi.org/10.1007/s40314-017-0530-1.

[24] M.A. Zaky, S.S. Ezz-Eldien, E.H. Doha, J.A. Tenreiro Machado and A.H. Bhrawy. An efficient operational matrix technique for multidimensional variableorder time fractional diffusion equations. Journal of Computational and Nonlinear Dynamics, 11(6):061002, 2016. https://doi.org/10.1115/1.4033723.

[25] M. Zayernouri and G.E. Karniadakis. Fractional spectral collocation methods for linear and nonlinear variable order FPDEs. Journal of Computational Physics, 293:312-338, 2015. https://doi.org/10.1016/j.jcp.2014.12.001. 